\section{Structural Analysis of Sydowic Acid by X-ray Diffraction}

\section{Keiichi Fukuyama, * Tomitake Tsukthara,* Yukiteru KaTSUBE, * Takashi HaMASAKI and Yuichi Hatsuda}

Faculty of Agriculture, Tottori University, Koyama, Tottori 680

* Faculty of Engineering, Tottori University, Koyama, Tottori 680

Received February 23, 1976

Sydowic acid, $\mathrm{C}_{15} \mathrm{H}_{20} \mathrm{O}_{4}$, was isolated from Aspergillus sydowi (Bainier et Sartory) Thom et Church, and its structure was shown to be I by the analysis of chemical and spectroscopic data. ${ }^{1 /}$ However, the $[\alpha]_{\mathrm{D}}^{21^{\circ}}$ value appearing in the previous paper is fairly small $\left(-6.4^{\circ}\right)$, hence it was questioned whether the metabolite is optically pure or not. In order to eliminate the ambiguity, and to obtain the stereochemistry of the metabolite, its structure has been investigated by the X-ray diffraction method.

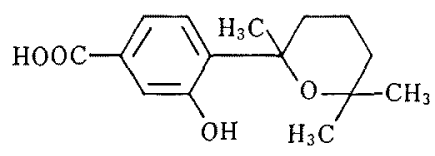

I

Crystals were obtained from acetone solution in the form of colorless prisms. They were triclinic, with two molecules in a unit cell of dimensions: $a=10.54, b=6.61, c=$ $11.01 \AA, \alpha=78.55, \beta=105.80, \gamma=96.78^{\circ}$. The intensities of 1744 independent reflections with $\sin \theta / \lambda<0.53$ were measured by stationarycrystal stationary-counter technique with $\mathrm{Ni}$ filtered $\mathrm{CuK} \alpha$ radiation. The intensities were corrected only for the Lorentz and polarization factors.

The structure was solved by the interpretation of Patterson function followed by successive Fourier syntheses. Since the arrangement of peaks in the Fourier map appeared to be centrosymmetric, the blockdiagonal least-squares refinement was made with $P \overline{1}$ space group hereafter. Oxygen atoms were identified on the basis of the isotropic temperature factors as well as chemical information. The refinement with anisotropic temperature factors for non-hydrogen atoms and isotropic ones for hydrogen atoms reduced the $R$ value to 0.10 for 1561 non-zero reflections. ${ }^{2}$ ?

The packing of molecules in a unit cell is shown in Fig. 1 by stereoscopic drawing. The present structural analysis has established that the metabolite is racemic compound or at least not optically pure. In the crystal, intramolecular hydrogen bonding is found between the phenolic hydroxyl group and ether oxygen atom. Intermolecular hydrogen bonds between carboxyl groups make a dimer around the center of symmetry. As shown in Fig. 1, the trimethyltetrahydropyrane ring takes the chair form.
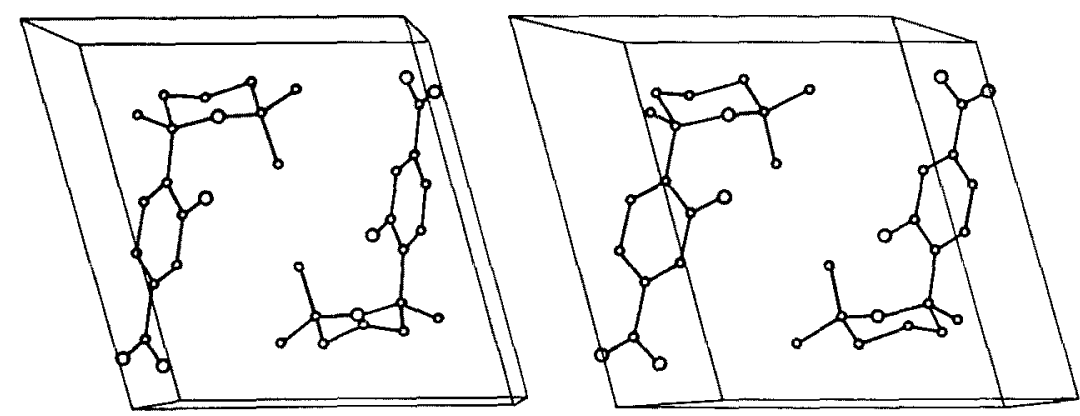

Frg. 1. Stereoscopic Illustration of the Molecular Arrangement in a Unit Cell.

The large and small circles indicate oxygen and carbon atoms respectively. The hydrogen atoms are omitted from the figure. 
Acknowledgement. The authors wish to thank Professor Tamaichi Ashida of Nagoya University for his permission to use the block-diagonal least-squares program. They also wish to thank Mr. Souei Furukawa and Mr. Atsuhiko Kunita for the X-ray measurement.

\section{REFERENCES}

1) T. Hamasaki, Y. Sato and Y. Hatsuda, Agr.
Biol. Chem., 39, 2337 (1975); T. Hamasaki, Y. Sato, Y. Hatsuda, M. Tanabe and L. W. Cary, Tetrahedron Letters, No. 9, 659 (1975).

2) The atomic scattering factors were taken from the "International Tables for X-ray Crystallography;" Vol. III, Kynoch Press, Birmingham, 1962, p. 202. 